\title{
Políticas de assistência estudantil em saúde nos institutos e universidades federais
}

\author{
Taís Bleicher \\ Universidade Federal do Ceará - Fortaleza - CE - Brasil \\ Raquel Campos Nepomuceno de Oliveira \\ Instituto Federal de Educação, Ciência e Tecnologia do Ceará - Jaguaribe - CE - Brasil
}

\begin{abstract}
Resumo
O trabalho aborda as políticas públicas de saúde estudantil das instituições federais de ensino técnico e superior, tendo como objetivo avaliálas. A partir de revisão bibliográfica, documental e da atuação das autoras, alguns impasses são levantados: ausência de ações conjuntas entre Ministério da Educação - MEC e Ministério da Saúde - MS; falta de capacitação de técnicos do MEC em Saúde Pública; segregação entre as ações de saúde estudantil e do trabalhador; falta de políticas para estudantes de pós-graduação; ações voltadas ao tratamento em Saúde, em detrimento das atividades de promoção, prevenção e articulação com a rede de Saúde; ausência de pesquisas que embasem as ações. Concluise que é necessária a criação de um modelo de serviço calcado na pesquisa, de acesso universal; e ações de prevenção e promoção em Saúde concernentes à realidade enfrentada nas instituições e por cada segmento do alunado.
\end{abstract}

Palavras-chave: Políticas Públicas; saúde; Ensino Superior.

\section{Policies in health assistance for students in federal institutes and universities}

\begin{abstract}
The article deals with the student health public policies of the federal institutions of technical and higher education, with the objective of evaluating them. Based on bibliographical, documentary and authorial review, some impasses are raised: absence of joint actions between the Ministry of Education - MEC and the Ministry of Health - MS; Lack of training of MEC technicians in Public Health; Segregation between student and worker health actions; Lack of policies for postgraduate students; Actions aimed at the treatment in Health, to the detriment of the activities of promotion, prevention and articulation with the Health network; Lack of research that supports the actions. It is concluded that it is necessary to create a service model based on universal access research; And prevention and promotion actions in Health concerning the reality faced in the institutions and by each segment of the student.
\end{abstract}

Keywords: Public Policies; health; Higher education.

\section{Políticas de asistencia estudiantil en salud en los institutos y universidades}

\section{federales}

\section{Resumen}

En este estudio se aborda las políticas públicas de salud estudiantil de las instituciones federales de enseñanza técnica y superior, teniendo como objetivo evaluarlas. A partir de revisión bibliográfica, documental y de la actuación de las autoras, algunos impases son levantados: ausencia de acciones conjuntas entre Ministerio de la Educación - MEC y Ministerio de la Salud - MS; falta de capacitación de técnicos del MEC en Salud Pública; segregación entre las acciones de salud estudiantil y del trabajador; falta de políticas para estudiantes de postgrado; acciones volcadas al tratamiento en Salud, en detrimento de las actividades de promoción, prevención y articulación con la red de Salud; ausencia de estudios que respalden las acciones. Se concluye que es necesaria la crianza de un modelo de servicio calcado en la investigación, de acceso universal; y acciones de prevención y promoción en Salud concernientes a la realidad enfrentada en las instituciones y por cada segmento del alumnado.

Palabras clave: Políticas Públicas; salud; enseñanza superior. 


\section{Introdução}

O processo de redemocratização brasileiro teve como marco a promulgação da Constituição Federal - CF - de 1988. Fruto de grandes embates políticos entre os movimentos sociais que visavam uma reforma de Estado e de grupos tradicionalmente poderosos, a CF instituiu oficialmente o sistema de seguridade social no Brasil, a partir do tripé Previdência, Saúde e Assistência Social. Era necessário criar políticas públicas que garantissem os direitos sociais. Até então, as políticas sociais brasileiras eram questionadas, caracterizadas como fragmentadas, excludentes e setorizadas. A CF, claramente progressista, buscava descentralizar os poderes e as funções do Estado. Os valores que perpassavam as diversas políticas públicas delineadas então eram a democratização e a participação. Quanto à democratização, a proteção social do Estado deveria ser universal, independente de sua contribuição ao sistema. Além disso, foram criadas diversas estruturas organizativas com vistas a possibilitar a participação popular, como conselhos, fundos, comissões e conferências A universalidade das políticas possibilitaria atender pessoas que não estavam vinculados ao mercado formal de trabalho e que, portanto, eram especialmente vulneráveis (Cunha \& Cunha, 2002).

A descentralização pôde ser concretizada pela adoção da modalidade de federalismo participativo.

A descentralização assumiu significados e conteúdos distintos - conforme o desenho de cada política específica, a distribuição prévia de competências e do controle sobre os recursos - entre os três níveis de governo. Descentralização podia significar transferência parcial ou total de responsabilidades do governo federal para os Estados; do governo federal para o nível local ou do governo estadual para o local. Podia significar, também, mudança de funções entre níveis de governo, ou de um nível do governo para outras organizações públicas ou privadas, por exemplo: do governo estadual para as escolas; ou do governo para organizações assistenciais, cooperativas, ONGs (Almeida \& Carneiro, 2003, p. 129)

Os anos 1990 foram ricos em produções legislativas e normativas ministeriais direcionadas a dar conteúdo e viabilizar os princípios constitucionais. "A tendência à descentralização impôs-se em todas as áreas de política social, exceto nas da previdência e ciência e tecnologia, que continuaram sob responsabilidade federal" (Almeida \& Carneiro, 2003, p. 128).

No âmbito da Saúde, buscava-se realizar os princípios de uma reforma baseada no cuidado entendido de forma integral, perpassando promoção, prevenção, tratamento, reabilitação, vigilância sanitária e epidemiológica, em um sistema público e universal. Para que isso acontecesse, seria necessário modificar a compreensão sobre o processo saúde-doença, entendendo-o a partir de seus determinantes políticos, sociais e econômicos. A municipalização significou a necessidade de criar novas formas de gestão, inclusive a financeira e criar e desenhar distintos equipamentos no- vos. Foi um momento rico, em que experiências inovadoras, como o Programa de Saúde da Família ou os Centros de Atenção Psicossocial foram criados, testados e recomendados como modelos nacionais.

Entretanto, os avanços ocorridos nos equipamentos ligados ao Ministério da Saúde não tiveram o mesmo ritmo que os serviços de saúde relacionados a outros ministérios, como, por exemplo, o Ministério do Planejamento, Orçamento e Gestão ou o Ministério da Educação. Especialmente, ressalte-se que o Ministério da Ciência e da Tecnologia permaneceu federalizado, o que necessitaria de um esforço administrativo maior para a articulação de ações de Saúde. Nos órgãos federais, tanto os serviços de saúde do trabalhador e os serviços de saúde estudantil continuaram desintegrados da rede sanitária, com ofertas basicamente concentradas em perícias e tratamentos pontuais. A saúde dos pesquisadores de pós-graduação ficou no limbo dos três ministérios, sem política própria de nenhum.

Foi somente no final da década 2000 que esse cenário começou a mudar. No caso da saúde do trabalhador, com a criação do Subsistema Integrado de Atenção à Saúde do Servidor - SIASS, que tem como público-alvo todos os servidores públicos federais. Para o SIASS, já existem claras diretrizes, metas de funcionamento, e foram oferecidas diversas capacitações para viabilizar o novo modelo de atuação. É objetivo deste trabalho avaliar o estado de uma política nacional de saúde do estudante do ensino federal.

\section{Metodologia}

Foi realizada uma revisão bibliográfica e documental sobre as políticas públicas brasileiras de Saúde e Educação, especialmente no que se refere à assistência estudantil. São levantadas questões próprias à atuação política e prática no campo, já que as duas autoras trabalharam na Assistência Estudantil: a primeira foi membro do grupo de trabalho de Saúde do Fórum Nacional de Pró-Reitores de Assuntos Comunitários e Estudantis - FONAPRACE e atua em uma universidade federal; enquanto a segunda foi coordenadora de Assuntos Estudantis em um campus do Instituto Federal de Educação, Ciência e Tecnologia do Ceará - IFCE.

\section{Contextualizando a criação de uma política de assistência estudantil para o estudante do ensino federal}

A crise pela qual passaram as universidades federais durante a gestão Fernando Henrique Cardoso foi suplantada por um movimento da recuperação de seu orçamento e por um importante processo de expansão e interiorização, no governo Luís Inácio Lula da Silva. Entretanto, além do investimento e da ampliação das estruturas, o governo Lula buscava fazer uma profunda reforma na educação superior, no nível da graduação, "para o aumento da qualidade dos cursos e pelo melhor aproveitamento da estrutura física e 
de recursos humanos existentes nas universidades federais, respeitadas as características particulares de cada instituição e estimulada a diversidade do sistema de ensino superior" (MEC, 2007a). Para isso, criou, em 2007, o Programa de Reestruturação e Expansão das Universidades Federais - REUNI. Um dos objetivos do REUNI era diminuir as taxas de evasão dos alunos. Para isso, seria necessário criar políticas de assistência estudantis voltadas para este propósito.

Assim, em 2007, através da Portaria Normativa $\mathrm{n}^{\circ}$. 39, do MEC (MEC, 2007b), o governo instituiu o Programa Nacional de Assistência Estudantil - PNAES, que foi implementado a partir de 2008. Em 2010, a portaria do MEC foi transformada em decreto presidencial de $n^{\circ} .7 .234$ (Decreto n. 7.234, 2010).

É verdade que, antes do PNAES, já existia repasse de recursos do Tesouro na Lei Orçamentária Anual para a assistência estudantil nas Instituições Federais de Ensino Superior - IFES, por meio do programa Brasil Universitário (Cislaghi \& Silva, 2013). O que não existia era diretrizes para uma política de assistência estudantil, o que permitia a configuração dos programas e ações a mercê do princípio da autonomia universitária.

Segundo o decreto (Decreto n. 7.234, 2010), os objetivos do PNAES seriam:

I - democratizar as condições de permanência dos jovens na educação superior pública federal;

II - minimizar os efeitos das desigualdades sociais e regionais na permanência e conclusão da educação superior;

III - reduzir as taxas de retenção e evasão; e

IV - contribuir para a promoção da inclusão social pela educação.

Como é óbvio, a atenção à Saúde não é objetivo das ações do MEC, mas sim, uma das formas de se conseguir chegar aos objetivos, especialmente, o de redução das taxas de evasão. Embora, em seu artigo $1^{\circ}$, o PNAES esteja claramente destinado à Educação Superior Pública, o seu artigo $4^{\circ}$ diz:

Art. 4ํ As ações de assistência estudantil serão executadas por instituições federais de ensino superior, abrangendo os Institutos Federais de Educação, Ciência e Tecnologia, considerando suas especificidades, as áreas estratégicas de ensino, pesquisa e extensão e aquelas que atendam às necessidades identificadas por seu corpo discente (Decreto n. $7.234,2010)$.

Em que pese a negligência do legislador em apontar os Institutos Federais - IF em seu artigo $1^{\circ}$, na prática, eles também estão regidos pelo PNAES, e os recursos são disponibilizados independentemente se se trata do ensino médio/ profissionalizante ou superior. Os pesquisadores estudantes de pós-graduação permaneceram em um vazio assistencial, em que não são considerados trabalhadores - não se beneficiam do SIASS - e nem são alvos das políticas de assistência estudantil - não se beneficiam do PNAES. Embora não haja ainda política de assistência estudantil específica direcionada a nenhum desses públicos, no primeiro caso a flexibilidade é usada para abranger, enquanto no segundo a definição da política é usada para excluir.

\section{Saúde do estudante do ensino público federal}

Vários problemas surgem a partir do PNAES, em particular no que diz respeito à atenção em Saúde. Ao contrário do SIASS, mesmo com o decreto do PNAES não houve um programa de capacitação dos técnicos quanto aos mecanismos de consecução dos objetivos da política. Os técnicos, ligados ao MEC, frequentemente não tinham formação ou perfil de atuação na Saúde. Como não há metas a serem cumpridas e nem mecanismos de avaliação da política, poucos gestores encamparam a necessidade da mudança de atuação, no sentido de voltar as ações para promoção e prevenção em saúde e integração dos serviços à rede municipal pública de Saúde. Nessa ausência de disposição e compreensão gerencial, o FONAPRACE - e a Associação Nacional de Pós-Graduandos - ANPG - têm sido atores políticos fundamentais para a construção de uma política de saúde para o estudante do ensino federal.

O FONAPRACE criou o seu grupo de trabalho nacional de saúde do estudante, a partir do I Seminário de Atenção à Saúde do Estudante, em 2012. O objetivo era discutir os princípios e diretrizes da assistência. No mesmo ano, a ANPG criou o seu Fórum de Pós-Graduandos em Saúde, durante o XXVII Congresso Nacional de Pós-Graduandos. Além disso, a ANPG tem sido ativa na luta para a inclusão dos pós-graduandos no PNAES.

Considerando estudos e pesquisas da última década (FONAPRACE, 2011; Cerchiari, Caetano, \& Faccenda, 2005; Assis, Sanabio, \& Machado, 2013; Almondes \& Araújo, 2003; Neves \& Dalgalarrondo, 2007; Louzada \& Silva Filho, 2005) que tratam do perfil de saúde dos estudantes de instituições federais, além dos dados já apresentados, o que se propõe a seguir são algumas reflexões a respeito do que já existe e, principalmente, das poucas tentativas iniciais de direcionamento da assistência estudantil nesta área.

Segundo o relatório do FONAPRACE (FONAPRACE, $2011)$, quase a metade dos estudantes $(41,7 \%)$ utiliza serviços públicos de saúde, especialmente os estudantes das classes C, D e E. Sendo esses os estudantes público-alvo das ações do PNAES, pode-se observar que a atenção à saúde é matéria contundente para a execução das ações relativas ao programa, na perspectiva de uma assistência estudantil que busca fugir do modelo assistencialista restrito a repasses financeiros aos estudantes.

No que concerne a essa área e de acordo com dados referentes ao relatório, Assis e cols. (2013) apontam que foram identificados os seguintes temas de especial atenção: prevenção de Doenças Sexualmente Transmissíveis [DST]/ 
AIDS, necessidade de intervenções em saúde mental, dependência química e programas de prevenção em saúde oral. Ainda segundo de estes autores, há recomendações específicas do FONAPRACE para ações vinculadas à área da saúde:

Entre as recomendações do FONAPRACE, no campo da saúde, destacam-se a necessidade de implantação, em caráter de urgência, de políticas de assistência integral à saúde dos estudantes, incluindo em caráter emergencial e prioritário programas de prevenção e redução de danos causados pelo uso indevido de álcool e outras drogas, e com objetivo de proporcionar qualidade de vida aos alunos (Assis \& cols., 2013, p. 09).

Focalizar as ações em saúde em intervenções de combate à dependência química, no entanto, parece ser um erro de diretriz do FONAPRACE. Segundo seu próprio relatório de perfil dos estudantes de graduação das universidades federais (FONAPRACE, 2011), "É baixa a proporção de estudantes que declara a interferência do uso abusivo de álcool $(4 \%)$ e de drogas não lícitas $(2 \%)$ em seu desempenho acadêmico" (p. 42). O perfil não faz nenhuma referência à dependência de drogas. Outro documento utilizado pelo FONAPRACE para referendar suas diretrizes é o I Levantamento Nacional sobre o Uso de Álcool, Tabaco e outras Drogas entre Universitários das 27 Capitais Brasileiras (Decreto n. 7.234, 2010), que é taxativa ao afirmar que, embora, quando comparados o segmento dos universitários à população geral, o uso de álcool seja maior entre os universitários, a dependência é maior entre a população que entre os universitários. Estes dados parecem apontar para o não conhecimento técnico sobre as diferenças entre uso, abuso e dependência de drogas. É possível se questionar sobre o conhecimento especializado em saúde que subsidia suas diretrizes. Especificamente quanto às atuações majoritárias em saúde na assistência estudantil, não há pesquisas que respaldem a necessidade específica de atuação de prevenção às DST neste público.

Um dos principais entraves apontados pelos autores é a falta de financiamento suficiente para atender à demanda nas instituições. As recomendações do FONAPRACE para minimizar essas dificuldades, no entanto, são contraditórias, pois centram as possíveis soluções nos gestores institucionais responsáveis pela Assistência Estudantil, e não no fortalecimento da política em nível nacional.

Mas há aqui uma questão que precede as conclusões a respeito da suficiência da verba frente à demanda. Se não há um delineamento claro para os investimentos financeiros em cada área proposta no PNAES, não há garantias de que haja equidade entre as áreas. O comum é a priorização de algumas dessas áreas, especialmente alimentação, transporte e moradia, em detrimento de outras também comprovadamente relevantes, como a saúde.

A ausência de regulamentação específica para as ações direcionadas pelo PNAES se, por um lado, permite que cada instituição proponha atividades adequadas às suas necessidades locais, por outro, não garante que haja atendimento às áreas estabelecidas no decreto, ou sequer um consenso geral a respeito de como tais atividades devem ser organizadas e promovidas no cotidiano institucional. Assim, pode ocorrer que, enquanto uma parcela das instituições direcione suas ações de saúde à promoção e prevenção articuladas à rede, outra parcela entenda que oferecer atendimento em saúde é oferecê-lo apenas em modelos de consultórios, semelhante a uma clínica particular com serviços individuais especializados.

Pesquisa realizada no Scholar Google com os termos "Saúde", "Estudante" e "Instituto Federal de Educação, Ciência e Tecnologia" não apresentaram nenhuma produção que tratasse da saúde deste alunado. Quando modificados os termos para "Saúde", "Estudante" e "Pós-Graduação", também não houve material sobre este público. É verdade que, com a modificação dos termos e substituição do termo "saúde" por "sofrimento", é possível encontrar uma pequena produção, sobre os estudantes de pós-graduação que poderia ser incluída no âmbito da saúde mental.

É possível defender que os IF têm uma trajetória recente, que data de 2007; enquanto que os pós-graduandos podem não contar com serviços de saúde uma vez que não se incluem no PNAES. Entretanto, as pesquisas existentes sobre saúde do estudante universitário, realizadas majoritariamente por professores e estudantes de pós-graduação, levam a acreditar que as equipes de técnicos dos IF e universidades federais não calcam suas ações por meio de pesquisas acadêmicas.

O entendimento de que os estudantes do ensino federal são especialmente vulneráveis a algumas formas de adoecimento, sobretudo em questões relacionadas à saúde mental, é a justificativa para a necessidade de criação de uma política pública específica.

\section{Saúde mental do estudante universitário}

Os estudos a respeito da saúde mental de estudantes universitários brasileiros são recentes, concentrando-se, sobretudo, da segunda metade da década de 1980 até os dias atuais. Esses estudos direcionam-se principalmente à incidência de transtornos mentais em estudantes universitários, com maior número voltado a estudantes da área da Saúde, cujo principal indicativo é a procura por serviços de atendimento psicológico e/ou psiquiátrico por parte dos estudantes. Poucos estudos são identificados a respeito do tema em outras áreas do conhecimento (Cerchiari \& cols., 2005).

De maneira geral, segundo revisão realizada por Cerchiari, Caetano e Faccenda (2005), os estudos têm apontado uma prevalência maior de transtornos mentais menores em populações universitárias que na população geral. Alguns fatores são apontados como correlatos à alta incidência desses transtornos em estudantes universitários, entre eles o excesso de atividades, que causa alteração do padrão de sono dos estudantes, gerando situações de ansiedade mais frequentes (Almondes \& Araújo, 2003); a perspectiva 
de futuro em relação à formação e ao papel social (Cerchiari \& cols., 2005) e especialmente a não-convivência com a família (Cerchiari \& cols.; Neves, 2007). O público feminino aparece como mais vulnerável à incidência de transtornos mentais em todos os textos pesquisados a respeito da saúde mental do estudante universitário.

Ainda de acordo com o relatório do Perfil Socioeconômico e Cultural dos Estudantes de Graduação das Universidades Federais Brasileiras (FONAPRACE, 2011) 55,28\% dos estudantes reside com os pais, e 5, $85 \%$ em casa de familiares; enquanto que o restante reside com amigos, outros familiares, casa mantida pela família, pensão, hotel, pensionato ou república. Se retomarmos as revisões já citadas (Cerchiari \& cols., 2005), que consideram vulneráveis os estudantes que durante a formação acadêmica permanecem distantes da família, esse é um dado relevante para que a criação de programas específicos de atenção a este público.

Os dados referentes a essa pesquisa foram coletados no ano de 2010. Do ano de 2009 ao ano de 2014, apenas no IFCE, foram criados ou autorizados 17 novos campi no interior do estado, como parte da concretização da expansão universitária proposta nos governos do PT. Ainda não há dados disponíveis referentes ao perfil atual do estudante de ensino superior federal, mas imagina-se que, com a expansão e interiorização universitária, o contingente de estudantes que se desloca do convívio familiar para fazer um curso superior seja ainda maior, o que poderá criar uma alta demanda para as equipes de assistência estudantil.

Estudos na área de saúde mental do estudante direcionados ao público de pós-graduação são pouco numerosos. No que tange ao sofrimento relacionado à formação do pesquisador, datam de 1970 os primeiros estudos internacionais sobre esse tema, mas ainda de maneira indiferenciada entre estudantes de graduação, pós-graduação e pesquisadores estabelecidos. No âmbito nacional, Louzada e Silva Filho (2005) apontam para o fato de que a atividade de pesquisa tem sido investigada frequentemente em temas como investimento em pesquisa; formação da comunidade científica, produtividade científica; perfil da ciência; financiamento de postdocs, pós-graduação e trabalho. Entretanto, apenas recentemente a formação dos pesquisadores da pós-graduação tem sido destaque.

Enfocando especificamente o sofrimento de pesquisadores, foi possível encontrar duas pesquisas brasileiras. A primeira, conduzida por De Méis, Velloso, Lannes, Carmo e De Meis (2003), partiu de entrevistas com pesquisadores estabelecidos e pós-graduandos da área de bioquímica e identificou a existência de síndrome de burnout naquele grupo e $21 \%$ das pessoas pesquisadas com pelo menos um atendimento psiquiátrico ou psicológico. Em suas conclusões esses autores afirmam que o crescimento da ciência brasileira se dá graças a um enorme desgaste emocional das pessoas envolvidas. A segunda, realizada por NogueiraMartins e cols. (2004), abordou, no âmbito da psiquiatria, 146 pós-graduandos (99 mestrandos e 47 doutorandos) em um centro de saúde mental da Universidade Federal de São
Paulo... As crises apresentadas foram classificadas em crises adaptativas ou psicopatológicas. As categorias diagnósticas mais frequentes foram: transtorno depressivo e ansioso. $\mathrm{O}$ artigo aponta ainda a possibilidade de identificação precoce de estudantes com distúrbios emocionais e a necessidade de encaminhamento para serviços especializados, que forneçam atenção estruturada e confidencial. Alertam também no sentido de que os orientadores e programas de pós-graduação estejam empenhados na construção de um ambiente de treinamento acolhedor (Louzada \& Silva Filho, 2005, p. 452).

No caso de Louzada e Silva Filho (2005), estes realizaram pesquisa sobre os relatos de sofrimento de mestrandos e doutorandos ante a atual organização da pós-graduação brasileira. Constataram que frequentemente esse sofrimento era naturalizado pelos estudantes, embora que, por vezes, fosse remetido às regras próprias ao campo científico. Nesse caso, as condições e relações de trabalho, as regras de fomento, a equipe de pesquisa, as colaborações estabelecidas; a posição do laboratório no campo; o nível de reconhecimento científico do orientador; a posição do programa de pós-graduação; o estilo do orientador eram fatores que podiam se associar ao grau de sofrimento do pós-graduando. Nesse sentido, os autores apontam para dois níveis de ação: um de nível institucional, que diz da capacidade de realizar uma escuta cuidadosa do sofrimento do pós-graduando, e outra que diz respeito à necessária modificação das políticas educacionais e de Ciência e Tecnologia no Brasil, no sentido de estarem dedicadas não só à produtividade, mas às singularidades do sujeito que realiza o trabalho científico. É interessante verificarmos que, de toda a bibliografia pesquisada, apenas este texto sobre pós-graduação aponta para a identificação do sofrimento psíquico pelo viés do entrevistado, e não apenas para os dados estatísticos a respeito do adoecimento do estudante.

Nossa hipótese é a de que este fato se dá por conta de ser este um campo de pesquisa novo, o que faz necessário, portanto, gerar dados para subsidiar as propostas de intervenção. Nesse sentido, embora o foco no adoecimento não seja a ideia defendida pelas autoras, este é um primeiro material para pautar as ações na área. A partir desses dados, é necessário que sejam elaboradas propostas de intervenção direcionadas à promoção e prevenção de saúde mental de acordo com as necessidades já identificadas.

Se compreendermos que as relações institucionais no ambiente educacional, como apontam Bacchereti e Molina (2012), são determinantes para o estabelecimento do processo de ensino e aprendizagem, e mais que isso, para a formação ampla do estudante, podemos concluir que essas relações têm forte impacto na saúde mental do público estudantil. Relações institucionais adoecedoras, portanto, contribuiriam para representações escolares também adoecedoras, impedindo, assim, o desenvolvimento pleno das atividades no ambiente educacional, na medida em que impactariam negativamente no rendimento e na formação do estudante. 
É justamente nesse aspecto que se pode ressaltar a relação do presente trabalho com a atuação em Psicologia Escolar e Educacional. Ainda de acordo com Bacchereti e Molina (2012), é papel do psicólogo na educação "realizar diagnósticos, análises, intervenções e pesquisas com base na descrição e análise das relações escolares e nos processos de subjetivação/objetivação dos indivíduos nele envolvidos". Como apontado anteriormente, não há dados suficientes para subsidiar as ações direcionadas aos diferentes públicos aos quais o PNAES se destina. Além disso, considera-se que o PNAES não atinge públicos sobre os quais há dados de demanda para assistência estudantil, como a pós-graduação. É justamente papel dos psicólogos atuantes nesses espaços do Ensino Superior direcionar suas ações de maneira a que os públicos atendidos o sejam adequadamente e que os públicos ainda não atendidos possam ter acesso às ações direcionadas às suas necessidades particulares.

Além de utilizar os dados já existentes para a elaboração de ações, é necessário que sejam realizadas pesquisas sistemáticas para identificar dados como: o perfil de estudantes de cada instituição, com seus respectivos níveis de ensino - e não apenas no que diz respeito à graduação; que parcela dos estudantes é atingida pelos programas da Assistência Estudantil; o perfil de saúde desses estudantes; que modelos de serviços direcionados à saúde do estudante existem atualmente na assistência estudantil. A identificação desses dados é o meio mais adequado para subsidiar a elaboração de um modelo que possa atender de maneira apropriada às exigências relativas a todo o corpo estudantil de cada instituição, e não apenas a parcelas deste. Nos casos em que ainda não há uma política específica, como os níveis de pós-graduação, esses primeiros dados têm justamente o intuito de deixar registrado que a demanda existe e que é necessária a ampliação da política atual de Assistência Estudantil ou a criação de uma política nova direcionada a esse público.

\section{Conclusões}

No campo político, embora a elaboração e a execução do PNAES sejam de fundamental importância no contexto da expansão e interiorização universitária propostas pelo REUNI, é ainda necessário que este trabalho passe por processos de sistematização que permitam a distribuição adequada dos financiamentos, de maneira a abranger todas as áreas propostas no Decreto $7.234 / 2010$, sobretudo no que diz respeito à atenção à saúde do estudante das IFES, público sobre o qual atualmente já há dados que demonstram uma situação especial de vulnerabilidade. É necessária a inclusão dos estudantes de pós-graduação no PNAES ou a criação de uma política de assistência estudantil própria, que considere a implicação do atual modelo de Ciência e Tecnologia no país e seu impacto sobre a saúde do pesquisador.
No campo técnico, faz-se urgente capacitar as equipes em Saúde, com ênfase às questões relativas à Saúde Pública. Realizar programas e ações a partir de prévio levantamento diagnóstico institucional e com a compreensão de que já existe uma rede pública de saúde, que deve se comunicar com os serviços de saúde estudantil das instituições federais. Desta maneira, as instituições atuariam no sentido de criar ambientes saudáveis e prevenir doenças associadas especificamente à realidade do aluno do ensino federal; enquanto que a rede local de saúde seria acionada quando da necessidade de tratamento.

No âmbito acadêmico, sugere-se o incentivo à realização de pesquisas, compreendendo que alunos das diferentes IFES são públicos distintos entre si, dadas as diferenças que compõem os alunados dos IFs e das universidades; que alunos de graduações universitárias e de pós-graduações são também públicos distintos e, portanto, cada público deve ter suas especialidades observadas na condução dos estudos.

\section{Referências}

Almeida, M. H. T. \& Carneiro, L. P. (2003). Liderança local, democracia e políticas públicas no Brasil. Opinião Pública, 9(1), 124-147.

Almondes, K. M. \& Araújo, J. F. (2003). Padrão do ciclo sono-vigília e sua relação com a ansiedade em estudantes universitários. Estudos de Psicologia, 8(1), 37-43.

Assis, A. C. L., Sanabio, M. T., Magaldi, C. A., \& Machado, C. S. (2013). As políticas de Assistência Estudantil: experiências comparadas em universidades públicas brasileiras. Revista Gestão Universitária na América Latina, 6(4), 125-146.

Bacchereti, S. F. \& Molina, R. (2012). Transitando pelo universo da escola: relações institucionais e processos de intervenção. Em R. Molina \& C. B. Angelucci (Orgs.), Interfaces entre psicologia e educação: Desafios para a formação do psicólogo (pp. 63-80). São Paulo: Casa do Psicólogo.

Cerchiari, E. A. N., Caetano, D., \& Faccenda, O. (2005). Prevalência de transtornos mentais menores em estudantes universitários. Estudos de Psicologia, 10(3), 413-420.

Cislaghi, J. F. \& Silva, M. T (2013). O Plano Nacional de Assistência Estudantil: Ampliação de Vagas X Garantia de Permanência. Revista SER Social, 14(31), 473-496.

Cunha, E. P. \& Cunha, E. S. M. (2003). Políticas Públicas e Sociais. Em A. Carvalho \& cols. (Orgs.), Politicas públicas (p. 11-25). Belo Horizonte: UFMG.

Decreto no. 7.234 (2010, 19 de julho). Dispõe sobre o Programa Nacional de Assistência Estudantil - PNAES. Recuperado: 27 jul. 2015. Disponível: http://www.planalto.gov.br/ccivil_03/_Ato20072010/2010/Decreto/D7234.htm. 
Fórum Nacional de Pró-Reitores de Assuntos Comunitários e Estudantis [FONAPRACE] (2011). Perfil Socioeconômico e Cultural dos Estudantes de Graduação das Universidades Federais Brasileiras. Brasília.

Louzada, R. C. R. \& Silva Filho, J. F. (2005). Formação do pesquisador e sofrimento mental: um estudo de caso. Psicologia em Estudo 10(3), 451-461.

Ministério da Educação [MEC] (2007a). REUNI. Reestruturação e expansão das universidades Federais. Diretrizes Gerais. Recuperado: 15 jan. 2015. Disponível: http://portal.mec.gov.br/ sesu/arquivos/pdf/diretrizesreuni.pdf.
Ministério da Educação [MEC] (2007b). Portaria Normativa $n^{\circ} .39$, de 12 de dezembro de 2007. Institui o Programa Nacional de Assistência Estudantil - PNAES. Recuperado: 15 jan. 2015. Disponível: http://portal.mec.gov.br/arquivos/pdf/portaria_pnaes. pdf.

Neves, M. C. C. \& Dalgalarrondo, P. (2007). Transtornos mentais auto-referidos em estudantes universitários. Jornal Brasileiro de Psiquiatria, 56(4), 237-244.

Recebido em: 29/07/2015

Reformulado em: 02/07/2016

Aprovado em: 07/07/2016

\section{Sobre as autoras}

Taís Bleicher (taisbleicher@hotmail.com)

Mestre em Psicologia Clínica e Cultura - UnB. Doutora em Saúde Coletiva - UFC/UECE/UNIFOR. Doutorado-sanduíche em Psicología Social - UCM, Espanha. Psicóloga da Universidade Federal do Ceará -

Raquel Campos Nepomuceno de Oliveira (quelcno1@gmail.com)

Psicóloga - UFC. Mestranda em Políticas Públicas e Gestão da Educação Superior - UFC. Psicóloga do Instituto Federal de Educação,

Ciência e Tecnologia do Ceará - Campus Jaguaribe.

Trabalho originalmente apresentado na Mostra de Ciência e Tecnologia da 9a Bienal da União Nacional dos Estudantes - UNE, sob coordenação da Associação Nacional de Pós-Graduandos - ANPG. 